\title{
Multi-scale interactions of geological processes during mineralization: cascade dynamics model and multifractal simulation
}

\author{
L. Yao ${ }^{1}$ and Q. Cheng ${ }^{1,2}$ \\ ${ }^{1}$ State Key Laboratory of Geological Processes and Mineral Resources, China University of Geosciences, \\ Wuhan 430074, China \\ ${ }^{2}$ Department of Earth and Space Science and Engineering, York University, Toronto, ON M3J1P3, Canada
}

Received: 3 January 2010 - Revised: 10 October 2010 - Accepted: 9 February 2011 - Published: 8 March 2011

\begin{abstract}
Relations between mineralization and certain geological processes are established mostly by geologist's knowledge of field observations. However, these relations are descriptive and a quantitative model of how certain geological processes strengthen or hinder mineralization is not clear, that is to say, the mechanism of the interactions between mineralization and the geological framework has not been thoroughly studied. The dynamics behind these interactions are key in the understanding of fractal or multifractal formations caused by mineralization, among which singularities arise due to anomalous concentration of metals in narrow space. From a statistical point of view, we think that cascade dynamics play an important role in mineralization and studying them can reveal the nature of the various interactions throughout the process. We have constructed a multiplicative cascade model to simulate these dynamics. The probabilities of mineral deposit occurrences are used to represent direct results of mineralization. Multifractal simulation of probabilities of mineral potential based on our model is exemplified by a case study dealing with hydrothermal gold deposits in southern Nova Scotia, Canada. The extent of the impacts of certain geological processes on gold mineralization is related to the scale of the cascade process, especially to the maximum cascade division number $n_{\max }$. Our research helps to understand how the singularity occurs during mineralization, which remains unanswered up to now, and the simulation may provide a more accurate distribution of mineral deposit occurrences that can be used to improve the results of the weights of evidence model in mapping mineral potential.
\end{abstract}

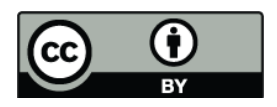

Correspondence to: Q. Cheng (qiuming@yorku.ca)

\section{Introduction}

Mineralization has complex connections with various geological processes and geologists can deduce part of these connections from outcrop exploration. For example, if certain deposits are always coexisting with a special type of intrusion, we may consider that this intrusion is related to mineralization during geological periods. The summarization of such relations between mineralization and certain geological processes and their geological characteristics leads to metallogenic models for distinct genetic types of mineral deposits (Cox and Singer, 1986). Metallogenic models give a comprehensive knowledge of favourable conditions for mineralization, but they are far too insufficient to capture the mechanism of the interactions between mineralization and the geological framework, because they are inherently descriptive. Such a mechanism is indispensable as we wonder why common fractal or multifractal features has been formed throughout mineralization.

A variety of approaches have already been proposed for quantifying and integrating the relations between mineralization and certain geological processes to evaluate mineral potential, but they have not explained the physical backgrounds of these relations, such as weights of evidence (Bonham-Carter et al., 1988; Cheng and Agterberg, 1999; Zhang et al., 2008), logistic regression (Sahoo and Pandalai, 1999), Bayesian network (Porwal et al., 2006), neural network (Singer and Kouda, 1996; Porwal et al., 2003; Nykänen, 2008), evidential belief functions (Carranza et al., 2005, 2008) and expert-guided methods (Cassard et al., 2008). Most of the methods are data-driven, that is to say, the dynamics between mineralization and certain geological processes are unstable and only empirically established

Published by Copernicus Publications on behalf of the European Geosciences Union and the American Geophysical Union. 
based on observations. Mineralization has been thought of as a self-organization process with dissipative structures and, thus, nonlinear features and complexities emerged (Yu et al., 1988), but the dynamical model of the process is too sensitive for the parameters that it is hard to characterise. A novel understanding of the mineralization is its interpretation as a singular event (Cheng, 2007b,a), which always results in multifractal products. Besides mineralization, typical singular events include hazard events, such as earthquakes, landslides, volcanoes, floods and hurricanes, because they result in anomalous amounts of energy release or mass accumulation confined to narrow intervals in space or time (Cheng, 2007b,a). However, the mechanism of mineralization as a singular event remains a puzzle.

Generally speaking, the dynamics of the interactions between mineralization and the geological framework is still lacking in systematic research. The answer to the above question would aid in understanding why singularity occurs and the multi-scale nature of mineralization. In this paper, we construct a cascade model to simulate interactions between mineralization and the relevant geological processes. Then, a case study of hydrothermal gold mineral deposits in southern Nova Scotia, Canada is described for multifractal simulation, in which we use some statistics from Cheng (2008). Through our research, we think that mineralization may be accompanied by diverse geological processes, which exerted their impacts in a cascade-like manner and their impacts are associated with mineralization in different scales.

\section{Multifractal and singularity}

Since the fractal concept was proposed (Mandelbrot, 1977, 1983), fractal or multifractal methods became powerful tools for identifying geological features associated with mineralization. Fractal or multifractal modelling play important roles in the quantifying of mineral deposits (Turcotte, 1996), characterising the spatial distribution of mineral deposits (Carlson, 1991; Agterberg et al., 1996; Raines, 2008) and mapping mineral-potential (Cheng et al., 1996; Ford and Blenkinsop, 2008). New methods based on the multifractal theory, such as concentration-area (C-A) method (Cheng et al., 1994), spectrum-area (S-A) method (Cheng et al., 2000) and the singularity mapping technique (Cheng, 2007a), were developed to identify geochemical anomalies and to map mineral potential (Cheng, 2007b; Cheng and Agterberg, 2009; Zuo et al., 2009a).

While many mathematical geologists are familiar with multifractal spectrum $f(\alpha)$ in the model of Evertsz and Mandelbrot (1992); Schertzer and Lovejoy (1991), they developed another multifractal model in geophysics based on codimension function $C(\gamma)$, where $\alpha$ and $\gamma$ represent Hölder exponent and field order, respectively. Cheng (1996) compared these two models. Here, we introduce the elementary notations and formulas based on the $f(\alpha)$ model.
Suppose a geometrical support with linear size $\epsilon$ was defined as $A(\epsilon)$, and $\mu[A(\epsilon)]$ represents a kind of measure on $A(\epsilon)$, i.e., the most common measures of this kind related to mineralization may be the average geochemical concentrations on areas at different scales, then the following powerlaw relationships exist in conditions of multifractal:

$\mu[A(\epsilon)] \propto \epsilon^{\alpha}$

$N_{\alpha}(\epsilon) \propto \epsilon^{-f(\alpha)} \quad(\epsilon \rightarrow 0)$

where $\alpha$ means "proportional to", $\alpha$ is singularity index, $N_{\alpha}(\epsilon)$ is the number of cells having singularity index $\alpha$ while $\epsilon$ approaches zero and $f(\alpha)$ is the multifractal spectrum. Equation (1) is scale-invariant because the singularity index $\alpha$ remains constant under all rescalings of the linear size $\epsilon$. If the size is changed from $\epsilon_{n-1}$ to $\epsilon_{n}$ for some scale $\lambda$, that is, $\epsilon_{n}=\lambda \epsilon_{n-1}$, and the measure is changed from $\mu\left[A\left(\epsilon_{n-1}\right)\right]$ to $\mu\left[A\left(\epsilon_{n}\right)\right]$, respectively, then the following can be obtained from Eq. (1):

$\mu\left[A\left(\epsilon_{n}\right)\right] / \mu\left[A\left(\epsilon_{n-1}\right)\right]=\lambda^{\alpha}$

Actually fractal or multifractal describes the scaleinvariant relationship of a kind of measure on another kind of measure. Thus, the above formulae can be extended to Lebesgue measure space, and the support can even be fractal itself (Falconer, 2003). In this study, posterior probabilities of mineral deposit occurrences on certain geological conditions and the probabilities of occurrences of the relevant geological occurrences are Lebesgue measures, so their relationships can be multifractal only if the nonlinear interactions exist between them.

If $A(\epsilon)$ is the volume in $D$-dimensional space, a fractal density (Cheng, 2008) can be deduced from Eq. (1) as:

$\rho[A(\epsilon)] \propto \mu[A(\epsilon)] / A(\epsilon) \propto \epsilon^{\alpha-D}$

where fractal density $\rho$ is analogous to density in the physical sense, but this density changes with scale and can approach infinity; the index $(\alpha-D)$ also corresponds to the concept of codimension meaning the differences between the dimensions of space and fractal (Schertzer and Lovejoy, 1987).

\section{Multiplicative cascade models and their multifractal effect}

Multiplicative cascade models provide mathematical outlines to quantify turbulent intermittency and other extremely irregular complexities (Schertzer and Lovejoy, 1987). Cascade dynamics are fundamental in geological and geophysical processes. Typical geophysical fields are thought to result from cascade processes (Lovejoy et al., 2001; Lovejoy and Schertzer, 2007). The De Wijs model, was proposed to analyse the concentration of ores (De Wijs, 1951) and 


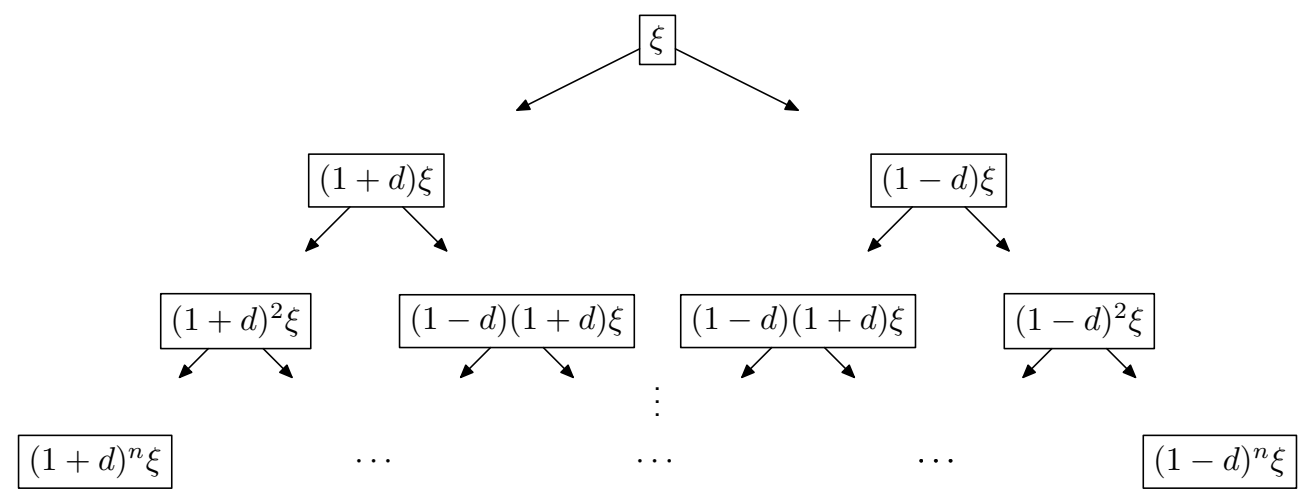

Fig. 1. 1-D multiplicative cascade model of De Wijs (De Wijs, 1951). The $\xi$ means original concentration value, and $d$ is the dispersion coefficient.

later it was adopted as a binomial cascade model to simulate multifractal (Mandelbrot, 1989). Various cascade models have been used to simulate rainfalls, clouds and geochemical concentrations (Deidda, 1999; Lovejoy and Schertzer, 2006; Agterberg, 2001).

A cascade process redistributes the mass concentrated on an support to small pieces through a series of repeated steps, leading to scale-invariant results. Taking the De Wijs model of geochemical distribution as an example, if the original concentration is $\xi$ and the support is 1 , then in the next iteration, the support is divided into two equal blocks of $1 / 2$ with a concentration of $(1+d) \xi$ and $(1-d) \xi$; here $d$ is the dispersion coefficient and is independent of block size; the procedure is iterated on the divided blocks (Fig. 1).

Generally considering a measure defined on a support, a basic cascade step includes two intertwining procedures: a partition procedure dividing current support into smaller parts while the scale drops down by a constant ratio; and a distribution procedure assigning different weights to the divided parts based on a probability distribution. The size of the ultimately divided supports would approach zero, while the cascade step is repeating endlessly. However, sometimes the cascade steps throughout a cascade process are limited and, thus, the ultimate size of the divided parts from the original support is determined by the divided times, which we denote as the maximum cascade division number $n_{\max }$ here. The measures allocated on the divided parts in each subdivision depend on the weights pattern for redistributing values from previous results. The maximum cascade division number $n_{\max }$ is meaningful to geological processes because it actually corresponds to the scale of a certain geological process. For example, the continental or ocean plate movement brings about global-scale change of geological settings, yet migrations of geochemical elements in a metallogenic province can only be on a regional-scale. To represent reality, many kinds of modifications on the basic model have been investigated. For example, Agterberg (2001) introduced minor disturbances in the cascade processes to simulate geo- chemical map patterns and proposed a random-cut variant of the De Wijs model (Agterberg, 2007a), Cheng (2005) applied a cascade model with variable partition processes, Schertzer and Lovejoy (1987) developed a cascade model continuous in scale.

The results of cascade dynamics are multifractal. The multifractal generated by this cascade process have many local maxima and minima with different singularities (Cheng, 2008). As a 1-D multiplicative cascade process, the minimal singularity $\alpha_{\min }$ and maximal singularity $\alpha_{\max }$ and their difference $\Delta \alpha$ can be calculated as:

$$
\begin{gathered}
\alpha_{\min }=1-\log _{2}(1+d) \\
\alpha_{\max }=1+\log _{2}(1+d) \\
\Delta \alpha=\alpha_{\max }-\alpha_{\min }=\log _{2} \frac{1+d}{1-d}
\end{gathered}
$$

where $d$ is the dispersion coefficient.

\section{Quantification of nonlinear dynamical interactions throughout mineralization}

\subsection{Statistical model of mineralization by weights of evidence}

Precisely characterising interactions throughout mineralization is almost impossible because of the limitation of data acquisition. Unlike other continuous measurements from some real-time instruments which are frequently used in meteorological or geophysical observatory, data related to mineralization and certain geological processes can only be acquired once from the present, since these processes have evolved for millions of years and there is no way to date them back. Continuous measurements are impossible and also meaningless in mineral exploration because the time scales are too large for us to find any changes of observation. The feasible way to quantify interactions between mineralization and the related 
geological processes comes from statistical analysis of various exploration data. The exploration data include observations from mineral deposits, geological structures, geochemical concentrations, petrological or stratigraphic formations and so on. A specific kind of exploration data actually represents products of a certain geological process, and the mineral deposits can be seen as results of mineralization. Therefore, analysing the relationships between mineralization and other kinds of exploration data provides an approximation to quantify the dynamical interactions between them. There are already models to utilize and integrate these relationships in order to evaluate mineral potential, as we have mentioned in the previous section. We choose the weights of evidence (WofE) method, which is very popular for mapping mineral potential, to statistically quantify the relationships between mineralization and certain geological processes, because it is reasonable and easy to understand.

The WofE model treats each kind of exploration data as a separate evidential layer and then integrates the evidential layers to support the final decision. Each kind of exploration data corresponds to the result of a certain geological event. In particular, the mineral deposit occurrences are products of mineralization. The geological events having a positive correlation with occurrences of mineral deposits are called favourable events in mapping mineral potential. Evidential layers are usually raster grids made of discrete cells to facilitate processing by GIS software in practice (Bonham-Carter, 1994). Each grid cell in an evidential layer falls into two distinct groups according to positive or negative correlation with occurrences of mineral deposits. Grid cells having positive correlation with occurrences of mineral deposits constitute favourable areas, and the remaining grid cells constitute unfavourable areas. Then the relationships between mineralization and certain geological events can be discussed by the posterior probabilities of mineral deposit occurrences on condition that these events occurred.

For simplicity, we use some specific symbols to represent formations of different geological events. We suppose the mineralization event as $D$ and, as a result, the prior probability of mineral deposit occurrence is known as $P(D)$; a favourable event to mineralization is denoted as $A$ and then the corresponding evidential layer is a binary pattern with $A$ or $\tilde{A}$ representing favourable events or not and, respectively, their probabilities of occurrences can be written as $P(A)$ and $P(\tilde{A})$. Thus, $D$ would be divided into $D A$ and $D \tilde{A}$, and the posterior probabilities can be represented by $P(D \mid A$ and $P(D \mid \tilde{A})$, respectively. From Bayesian rule, the posterior probabilities can be calculated as

$$
\begin{gathered}
P(D \mid A)=\frac{P(D) P(A \mid D)}{P(A)} \\
P(D \mid \tilde{A})=\frac{P(D) P(\tilde{A} \mid D)}{P(\tilde{A})} .
\end{gathered}
$$

If evidential patterns representing favourable geological events to mineralization are arranged as $A_{1}, A_{2}, \ldots, A_{n}$, while the contrary complements are denoted as $\tilde{A}_{1}, \tilde{A}_{2}, \ldots, \tilde{A}_{n}$, then a more comprehensive relation can be derived as

$$
\begin{aligned}
& P\left(D \mid A_{1} A_{2} \ldots A_{n}\right)=\frac{P(D) P\left(A_{1} \mid D\right) P\left(A_{2} \mid D\right) \ldots P\left(A_{n} \mid D\right)}{P\left(A_{1}\right) P\left(A_{2}\right) \ldots P\left(A_{n}\right)} \\
& P\left(D \mid \tilde{A_{1}} \tilde{A_{2}} \ldots \tilde{A_{n}}\right)=\frac{P(D) P\left(\tilde{A_{1}} \mid D\right) P\left(\tilde{A_{2}} \mid D\right) \ldots P\left(\tilde{A_{n}} \mid D\right)}{P\left(\tilde{A_{1}}\right) P\left(\tilde{A_{2}}\right) \ldots P\left(\tilde{A_{n}}\right)} .
\end{aligned}
$$

Note the Eqs. (7) require conditional independence assumption, which can be written as

$$
\begin{gathered}
P\left(A_{1} A_{2} \ldots A_{n} \mid D\right)=P\left(A_{1} \mid D\right) P\left(A_{2} \mid D\right) \ldots P\left(A_{n} \mid D\right) \\
P\left(\tilde{A_{1}} \tilde{A_{2}} \ldots \tilde{A_{n}} \mid D\right)=P\left(\tilde{A_{1}} \mid D\right) P\left(\tilde{A_{2}} \mid D\right) \ldots P\left(\tilde{A_{n}} \mid D\right) .
\end{gathered}
$$

The weights $W^{+}$and $W^{-}$are then defined as follows (Agterberg et al., 1990):

$$
\begin{aligned}
W_{0} & =\log _{\mathrm{e}}\{P(D) / P(\tilde{D})\} \\
W_{A}^{+} & =\log _{\mathrm{e}}\{P(A \mid D) / P(A \mid \tilde{D})\} \\
W_{A}^{-} & =\log _{\mathrm{e}}\{P(\tilde{A} \mid D) / P(\tilde{A} \mid \tilde{D})\} .
\end{aligned}
$$

Under the transformation of posterior probabilities by logit function, the logits of posterior probabilities of mineral deposit occurrences associated with multiple layers of evidence can be derived from Eqs. (9) assuming that conditional independence is satisfied:

$$
L\left(D \mid A_{1} A_{2} \ldots A_{n}\right)=W_{0}+W_{A_{1}}^{+}+W_{A_{2}}^{+}+\ldots+W_{A_{n}}^{+}
$$

where $L$ represents logit function and $L\left(D \mid A_{1} A_{2} \ldots A_{n}\right)$ means logit of $P\left(D \mid A_{1} A_{2} \ldots A_{n}\right)$. The similar formulae can be derived under other combinations of evidential layers. The logit function is important to logistic regression and the logit of a number $p$ between 0 and 1 is given by the formula:

$$
L(p)=\log \left(\frac{p}{1-p}\right) .
$$

Hence, the posterior probabilities of mineral deposit occurrences can be easily calculated by the inversion of the logits. However, most of the time the conditional independence of evidential layers is not met, so that the estimation of posterior probabilities may be biased. Reasonable adjustments to the posterior probabilities can improve this situation, but it needs more accurate knowledge of the distributions. We will later discuss the possible application of our research to deal with this problem. 


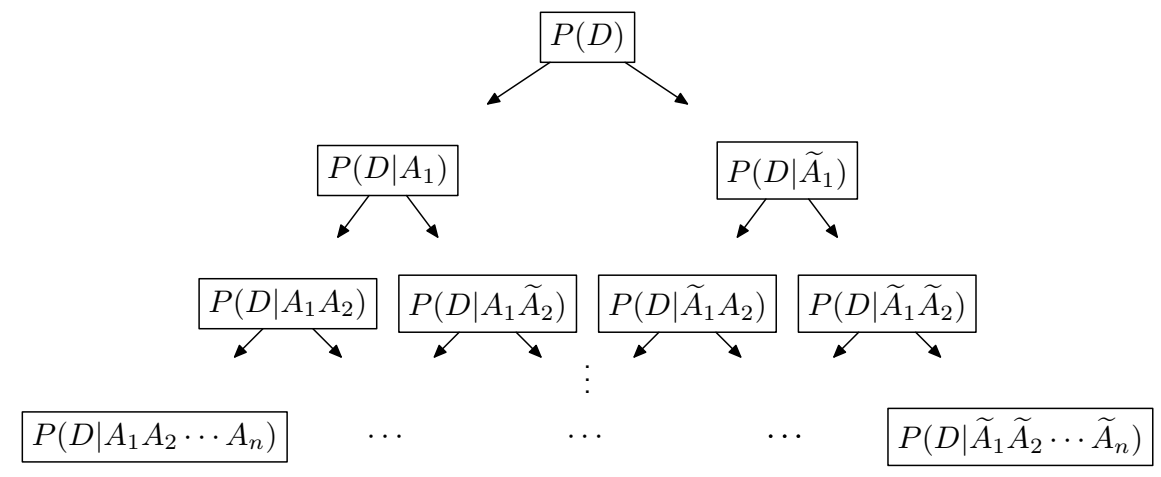

Fig. 2. Updating and integrating of evidential patterns in weights of evidence method. $P(D)$ means the prior probability of mineral deposit occurrence; $A_{1}, A_{2}, \ldots, A_{n}$ are different evidential patterns having positive correlations with mineral deposits and $\tilde{A}_{1}, \tilde{A}_{2}, \ldots, \tilde{A}_{n}$ are evidential patterns in contrary. The posterior probabilities are updated as the new evidence patterns are integrated; this figure can be compared with Fig. 1.

\subsection{Generalized cascade process throughout mineralization and singularities}

The similarity between the WofE method and multiplicative cascade models has been discussed in Cheng (2008), and a variant of WofE based on singularity was proposed as a novel approach for information integration. The posterior probabilities of mineral deposit occurrences are updated as new evidential patterns are put in (Fig. 2). Each evidential pattern represents the product of a certain geological process and the updated posterior probabilities represents its impact exerted on mineralization. Assuming mineralization as a singular event, the impacts caused by the participation of certain geological processes should be accumulated nonlinearly so that singularities emerge. The updating of each evidential pattern and the relevant posterior probabilities is very similar to one cascade step and, therefore, we take the successive participating of new evidential patterns as a generalized cascade process. The nonlinear dynamics of interactions throughout mineralization can then be constructed by a generalized cascade model.

For an arbitrary evidential layer A, suppose $A$ or $\tilde{A}$ means presence or absence of $A$, respectively. If we have $n$ evidential layers, then the total area can be divided into $2^{n}$ sub-areas at last, marked by the different combinations of evidence. On each iteration of adding an evidence layer, the posterior probability on each evidential combination will be distributed into two partitions. So this process can be regarded as a binary cascade process and the comparisons are shown in Fig. 3. As the figure shows, the singularity caused by the evidence layer A can be easily derived from Eq. (3) as:

$\alpha(A)=\log P(A \mid D) / \log P(A)$

The singularity of pattern $\tilde{A}$ can be defined from the same procedures as:

$\alpha(\tilde{A})=\log P(\tilde{A} \mid D) / \log P(\tilde{A})$.
Equations (12) and (13) are important formulae to characterise the singularities of dynamical interactions between mineralization and certain geological processes. The formulae are based upon the assumption of mineralization as a singular event and more detailed descriptions can be seen in Cheng (2008).

\section{Cascade dynamics model of mineralization through multifractal simulation}

Considering the posterior probability of mineralization due to a certain geological process as an indirect measurement of interaction between them, we can study the cascade dynamics of mineralization from statistical points of view. The dispersion coefficient $d$, in an ordinary de Wijs model, could be regarded as a random variable $D^{*}$ with a distribution of $P\left(D^{*}=d\right)=P\left(D^{*}=-d\right)=1 / 2($ Agterberg, 2007a). Here the distribution of $d$ should be identical to the distribution of an evidence which is denoted as $P_{\mathrm{e}}$ later on. The frequency distribution of concentration values generated by a 1-D multiplicative cascade model is logbinomial, that is, the logarithmically transformed values have binomial distribution (Agterberg, 2007a). So the logarithmic variance $\sigma^{2}$ can be derived as:

$$
\begin{aligned}
\eta & =(1+d) /(1-d) \\
\sigma^{2} & =n_{\max } P_{\mathrm{e}}\left(1-P_{\mathrm{e}}\right)(\ln \eta)^{2}
\end{aligned}
$$

where $n_{\max }$ means the maximum cascade division number. When $P_{\mathrm{e}}=1 / 2$, Eq. (14) will follow into $\sigma^{2}=n_{\max }(\ln \eta)^{2} / 4$ corresponding to a simple de Wijs model (Agterberg, 2007a). The variance can be estimated from its relationship with the logarithmic variance:

$$
\left.\frac{s(\ln x)}{s(x)} \approx \frac{d(\ln x)}{d x}\right|_{x=\bar{x}}=\frac{1}{\bar{x}} .
$$




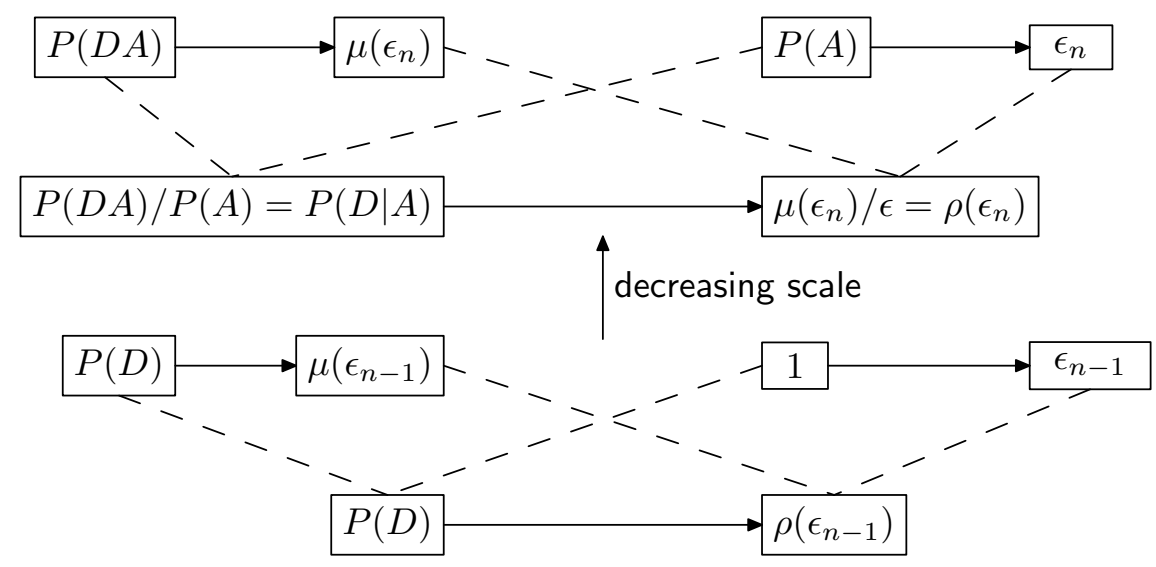

Fig. 3. Analogy between probabilities updated by an evidential layer and scale-invariant measures generated by multiplicative cascade process. This figure shows two consecutive iterations for demonstration. The prior probability $P(D)$ is defined on the whole support 1 , and one of its branches goes to posterior probability $P(D \mid A)$ on pattern $A$; this process is similar to the changing of measure from $\mu\left(\epsilon_{n-1}\right)$ to $\mu\left(\epsilon_{n}\right)$ as the scale decreases from $\epsilon_{n-1}$ to $\epsilon_{n}$, so does the fractal density $\rho$.

Equation (15) provides an approximation of variance for any random variable $x$ with mean $\bar{x}$ (cf. Agterberg et al., 1990). If the original concentration value in the multiplicative cascade model is $\xi$, then the variance of final concentration values should be:

$\beta^{2}=\xi^{2} \sigma^{2}$

where $\beta^{2}$ and $\sigma^{2}$ represent, respectively, variance and logarithmic variance of the final distribution. Therefore, we can deduce the maximum cascade division number $n_{\max }$ as long as the dispersion coefficient $d$ and logarithmic variance $\sigma^{2}$ or variance $\beta^{2}$ are known. The parameters $d$ and $n_{\max }$ are crucial parameters for the multiplicative cascade model and they have been applied to simulate geochemical distribution (Agterberg, 2007a,b).

Consider a certain evidence layer $A$, the dispersion coefficient $d$ can be estimated based on singularity calculated from Eqs. (12) and (13) as:

$\eta=\frac{1+d}{1-d}=2^{\alpha(A)-\alpha(\tilde{A})}$.

Equation (10) shows that the logits of posterior probabilities are the sum of weights determined by the combinations of all of the evidential patterns. The posterior probabilities stop updating after all the evidential layers are integrated successively and their results can be calculated from inversion of the logits. We suppose the posterior probabilities and their logits produced by WofE after integration of all available evidential layers are denoted as $P_{f}$ and $L_{f}$, respectively. From the definition of logit function, $P_{f}$ can be calculated as:

$P_{f}=e^{L_{f}} /\left(e^{L_{f}}+1\right)$.

The standard deviation of $P_{f}$ can be estimated by multiplying the standard deviation of $L_{f}$ by $P_{f}\left(1-P_{f}\right)$ (Fisher,
1971; Agterberg and Cheng, 2002). In order to estimate the standard deviation of $L_{f}$, firstly, variances of the weights can be estimated based on the asymptotic theory of discrete multivariate analysis (Agterberg et al., 1990); these can be augmented by variances for missing data and added to the variance of the prior logit, and then the result is an estimate of the standard deviation of $L_{f}$ (Agterberg and Cheng, 2002). So the variance of $P_{f}$ can be expressed as follows:

$s^{2}\left(P_{f}\right) \approx\left\{P_{f}\left(1-P_{f}\right) s\left(L_{f}\right)\right\}^{2}$

where $P_{f}$ and $L_{f}$ are the post probabilities of mineral deposit occurrences and their logits, respectively; $s^{2}\left(P_{f}\right)$ and $s^{2}\left(L_{f}\right)$ are the variances of $P_{f}$ and $L_{f}$, respectively.

If the total number of evidential layers is $m$ ( $m=4$ in this example), that is, the number of involved geological processes is $m$, then $2^{m}$ different patterns can be achieved by combination. The posterior probabilities would have $2^{m}$ possible values, so we can define a new random variable $P_{F}$ here and regard $P_{f}$ as the samples. Note that a binomial cascade model is used to distribute the posterior probabilities, so the expectation of $P_{F}$ should be equal to the prior probability $P_{0}$, which is considered as the original concentration. The probability of $P_{F}=P_{f}$ can be roughly estimated as the proportion of the pattern occupied by the $P_{f}$ in the total study area, thus, the variance of $P_{F}$ can be estimated as follow:

$s^{2}\left(P_{F}\right)=\frac{1}{n_{t}} \sum_{f=1}^{2^{m}} n_{f} s^{2}\left(P_{f}\right)$

where $n_{f}$ and $n_{t}$ are numbers of cells inside patterns occupied by $P_{f}$ and the total study area, respectively; $m$ means the number of evidential layers. Suppose the final probabilities satisfy logbinomial distribution as the cascading result, the variance of $P_{F}$ should be:

$s^{2}\left(P_{F}\right) \approx P_{0}^{2} \sigma^{2}$ 
Table 1. Statistics obtained from each of the four layers of binary maps (cf. Cheng, 2008).

\begin{tabular}{lllllllll}
\hline Layer & $P_{\mathrm{e}}$ & $\alpha$ & $\alpha(\sim)$ & $\Delta \alpha$ & $W^{+}$ & $s\left(W^{+}\right)$ & $W^{-}$ & $s\left(W^{-}\right)$ \\
\hline A & 0.394 & 0.276 & 2.960 & 2.684 & 0.67 & 0.25 & -0.97 & 0.47 \\
B & 0.568 & 0.084 & 3.657 & 3.573 & 0.57 & 0.23 & -2.23 & 1.00 \\
C & 0.398 & 0.125 & 4.370 & 4.245 & 0.81 & 0.24 & -1.68 & 0.67 \\
E & 0.194 & 0.391 & 3.465 & 3.074 & 1.00 & 0.31 & -0.53 & 0.32 \\
Average & 0.388 & 0.219 & 3.613 & 3.394 & & & & \\
\hline
\end{tabular}

where $P_{0}$ means the prior probability of mineral deposit occurrences. Finally, we derive the following equation based on Eqs. (14), (20) and (21):

$$
\begin{aligned}
n_{\max } & =\frac{s^{2}\left(P_{F}\right)}{P_{0}^{2} P_{\mathrm{e}}\left(1-P_{\mathrm{e}}\right)(\ln \eta)^{2}} \\
& =\frac{1}{n_{t}} \sum_{f=1}^{2^{m}} \frac{n_{f} P_{f}^{2}\left(1-P_{f}\right)^{2} s^{2}\left(L_{f}\right)}{P_{0}^{2} P_{\mathrm{e}}\left(1-P_{\mathrm{e}}\right)(\ln \eta)^{2}} .
\end{aligned}
$$

where $n_{t}$ and $n_{D}$ mean numbers of units of the total study area and units of the mineral deposits, respectively. Equation (22) can be used to estimate the maximum cascade division number $n_{\max }$.

\section{Case study: validating the cascade dynamics model of mineralization}

The nonlinear dynamics throughout mineralization were lacking research because of the difficulties to quantifying the interactions during mineralization. We propose a cascade dynamics model to characterise mineralization based on statistical analysis of exploration data by WofE. Here, we will use a case study to demonstrate the usage and validate this model. The case study simulates mineral potential of gold deposits in the southwestern Nova Scotia, Canada. The simulation is implemented on the basis of the cascade dynamics model of mineralization. About 20 gold deposit occurrences are found in sedimentary rocks in the study area of about $7780 \mathrm{~km}^{2}$. The study area is gridded into $1 \times 1 \mathrm{~km}^{2}$ cells as GIS map layer and four deliberately designed evidence layers were used in Cheng (2008). Layers A and B represent binary patterns determined by optimum distance from anticline axes $(2.5 \mathrm{~km})$ and optimum distance from the contacts between Goldenville and Halifax formations ( $4 \mathrm{~km}$ ), respectively. Layers $\mathrm{C}$ and $\mathrm{E}$ are two geochemical anomaly maps created by multifractal filter mapping of loadings of elements on two components derived via principle components analysis of geochemical concentrations of elements. In terms of geological backgrounds, layers A and B are, respectively, used to characterise the impacts to mineralization caused by the fold tectonics and metamorphosed sedimentary rocks which are subdivided into the Goldenville and Halifax formations; layers $\mathrm{C}$
Table 2. Statistics obtained from combinations of the four layers of binary maps (cf. Cheng, 2008). The digits 0 and 1 correspond to the presence and absence of binary patterns, respectively.

\begin{tabular}{lrllll}
\hline ABCE & $\begin{array}{r}\text { Area } \\
\left(\mathrm{km}^{2}\right)\end{array}$ & $n_{f} / n_{\mathrm{t}}$ & $L_{f}$ & $s^{2}\left(L_{f}\right)$ & $P_{f}$ \\
\hline 0000 & 2944.8 & 0.37851 & -11.37 & 1.8223 & 0.0000115 \\
0001 & 12.6 & 0.00162 & -9.84 & 1.816 & 0.0000533 \\
0010 & 333.8 & 0.04291 & -8.88 & 1.431 & 0.0001391 \\
0011 & 74.7 & 0.00960 & -7.35 & 1.4247 & 0.0006422 \\
0100 & 792.4 & 0.10185 & -8.57 & 0.8752 & 0.0001897 \\
0101 & 85.4 & 0.01098 & -7.04 & 0.8689 & 0.0008754 \\
0110 & 654.9 & 0.08418 & -6.08 & 0.4839 & 0.0022830 \\
0111 & 257.1 & 0.03305 & -4.55 & 0.4776 & 0.0104567 \\
1000 & 446.1 & 0.05734 & -9.73 & 1.6639 & 0.0000595 \\
1001 & 55.1 & 0.00708 & -8.2 & 1.6576 & 0.0002746 \\
1010 & 301.4 & 0.03874 & -7.24 & 1.2726 & 0.0007168 \\
1011 & 292.3 & 0.03757 & -5.71 & 1.2663 & 0.0033017 \\
1100 & 241.6 & 0.03105 & -6.93 & 0.7168 & 0.0009770 \\
1101 & 106.7 & 0.01371 & -5.4 & 0.7105 & 0.0044963 \\
1110 & 554.1 & 0.07122 & -4.44 & 0.3255 & 0.0116584 \\
1111 & 626.9 & 0.08058 & -2.91 & 0.3192 & 0.0516614 \\
\hline
\end{tabular}

and $\mathrm{D}$, respectively, correspond to the regional anomalies and local mineralization-associated anomalies favourable to mineralization which may originated from geochemical migrations in different scale (Cheng, 2008). More detailed descriptions of geological settings and datasets in this area can be found in $\mathrm{Xu}$ and Cheng (2001). For convenience, we only use some statistical results (Table 1) for our simulation from Cheng (2008) without discussing how to utilize the original data in the WofE method; more details about data processing and information integration can be found in Cheng (2008).

The value of $P_{\mathrm{e}}$ is set to the average probabilities of four patterns $\left(P_{\mathrm{e}}=0.388\right)$; the dispersion coefficients $d$ are calculated on the average range of singularity of each evidential layer and the value is $d=0.826$ (Table 1 ); the variance of logit $L_{f}$ equals the sum of variances of weights and variance of prior logit (Table 2). According to Eq. (22) and statistics from Table 2, the maximum cascade division number $n_{\max }$ is calculated as $n_{\max }=9.16 \approx 9$. The prior probability 


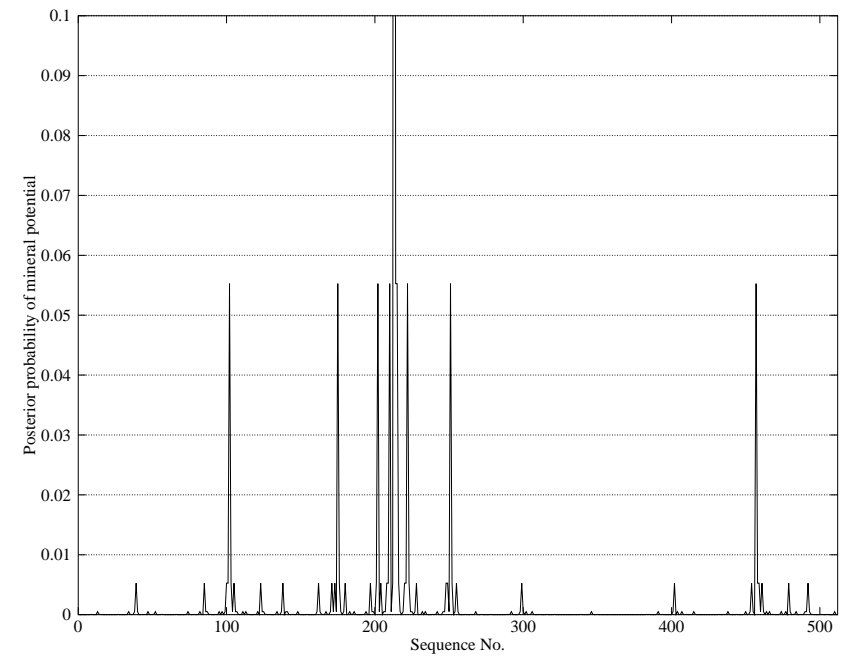

Fig. 4. Multifractal simulation of posterior probabilities of mineral deposit occurrences based on multiplicative cascade model; the original concentration was set to the prior probability of mineral deposit occurrences, and the highest value was truncated below 0.1 in the figure. The sequence numbers only represent an arbitrary ranking of the simulated results, and the maximum sequence number is determined by the maximum cascade number $n_{\max }$ as $2^{n_{\max }}$, that is, 512 in this example.

$P_{0}=20 / 7780=0.00257$ was set as the original concentration and distributed to the final partitions. Figure 4 shows the distribution of the posterior probabilities of mineral deposit occurrences generated by multifractal simulation, and the highest value was truncated below 0.1. Known from Eq. (10), the WofE method can only generate posterior probabilities of mineral deposit occurrences whose amount is no more than the number of combinations by the evidential layers. For example, the number of the values of posterior probabilities would not exceed $2^{m}$ if there are $m$ evidential layers involved. This sparse result provides little information for studying the distribution of mineral deposit occurrences. Although parts of statistical data are analysed from WofE, the respective mechanism represented by the cascade dynamics model is different from WofE. Thus, the distribution of the mineral potential generated by simulation is different from WofE. The number of possible results is associated to the maximum cascade division number $n_{\max }$, which reaches to $2^{n_{\max }}$ as Fig. 4 shows. However, the highest posterior probabilities which represent the most favourable areas to mineralization should be reflected in the result of multifractal simulation. From the simulation, we can see several peaks of values between 0.05 and 0.06 excluding one highest value; this is in accordance with the result of WofE. The multifractal results can also be used to explain why singularities occur throughout mineralization, of which dynamical mechanism are still not clear.

\section{Discussions and conclusions}

Mineralization is a long process, so that it is impossible to observe all the stages. However, nonlinear features have been discovered in the products of mineralization, from regionalscale of mineral deposits (Carlson, 1991; Agterberg et al., 1996), to micro-texture of minerals (Zhang et al., 2001; Zuo et al., 2009b), indicating that mineralization is a typical nonlinear process. In this article, we researched the interactions between mineralization and certain geological processes through statistical analysis of explorative data. Based on the proposition of taking mineralization as a singular event, the interactions can be regarded as generalized cascade dynamics and a cascade model was constructed to simulate the probabilities of mineral potential. Although the result is from a statistical point of view, cascade dynamics may be the physical nature of interactions between mineralization and certain geological processes. The maximum cascade division number $n_{\max }$ actually relies on the scale of the impacts of certain geological processes. Some factors, like regional tectonics, could have large scale impacts on mineralization, while some factors, like migration of geochemical elements, have finer scale impacts.

The research established a simple theoretic model to learn nonlinear dynamics throughout mineralization, and the exploration data are usually abundant to obtain so that the model is easy to set up. The multifractals generated by the cascade dynamics can be used to explain the singularities caused by mineralization. Unfortunately, the parameters in our model are also affected by conditional dependence of geological data. From Eq. (22), we can find that conditional dependence between evidence layers will increase the variance and lead to the larger $n_{\max }$. However, if cascade dynamics were true in mineralization, then we could have some empirical values of $n_{\max }$, and actually this type of $n_{\max }$ has been discussed in the geochemical distributions (Agterberg, 2007a). The empirical values of $n_{\max }$ should most probably comes from the stochastic distribution of mineral deposits from some mature exploration area, where good training sets can be ensured. Thus, we can give a rough estimation of probabilities of mineralization from the multifractal simulation and improve the conditional independence limitation of the weights of evidence method. It should be pointed out that the random generation of the dispersion coefficient $d$ is also important to the cascade model, which was simplified to an ordinary De Wijs model in this paper. Agterberg (2007a) proposed random generation of the normal distribution in simulating geochemical distributions but the applications were still not enough, and it may need further research in the future.

Acknowledgements. This article was revised on the presentation at IAMG'09, Stanford. The authors are in debted to Frits Agterberg for his kindness to provide some important references and programmes. The English writing was greatly improved with the help of Renguang Zuo from China University of Geosciences and 
Frits Agterberg. Thanks are due to two anonymous reviewers for their critical comments, and Ana Maria Tarquis for her careful review. The research was sponsored by a Distinguished Young Researcher Grant (40525009), a Strategic Research Grant (40638041), a General Research Grant (40972205) awarded by National Natural Science Foundation of China, a MOST Special Fund from the State Key Laboratory of Geological Processes and Mineral Resources, China University of Geosciences, a National High-tech R\&D Program of China (2009AA06Z110), and an Outstanding Youth Teacher Fund from CUG (CUGQNL0939).

Edited by: J. Davidsen

Reviewed by: E. J. M. Carranza, A. M. Tarquis, and another anonymous referee

\section{References}

Agterberg, F.: New applications of the model of de Wijs in regional geochemistry, Math. Geol., 39, 1-25, doi:10.1007/s11004-0069063-7, 2007a.

Agterberg, F. and Cheng, Q.: Conditional independence test for weights-of-evidence modelling, Natural Resources Research, 11, 249-255, doi:10.1023/A:1021193827501, 2002.

Agterberg, F., Bonham-Carter, G., and Wright, D.: Statistical pattern recognition for mineral exploration, in: Computer Applications in Resource Estimation, edited by: Gall, G. and Merriam, D., Pergamon, Oxford, 1-21, 1990.

Agterberg, F., Cheng, Q., and Wright, D.: Fractal modelling of mineral deposits, in: Proceedings of the International Symposium on the Application of Computers and Operations Research in the Minerals Industries, edited by: Elbrond, J. and Tang, X., Montreal, Canada, 43-53, 1996.

Agterberg, F. P.: Multifractal simulation of geochemical map patterns, J. China. Univ. Geosci., 12, 31-39, 2001.

Agterberg, F. P.: Mixtures of multiplicative cascade models in geochemistry, Nonlin. Processes Geophys., 14, 201-209, doi:10.5194/npg-14-201-2007, 2007 b.

Bonham-Carter, G.: Geographic Information Systems for Geoscientists: Modeling with GIS, Pergamon, Oxford, 1994.

Bonham-Carter, G., Agterberg, F., and Wright, D.: Integration of geological datasets for gold exploration in Nova Scotia, Photogramm. Eng. Rem. S., 54, 1585-1592, 1988.

Carlson, C. A.: Spatial distribution of ore deposits, Geology, 19, 111-114, 1991.

Carranza, E., Woldai, T., and Chikambwe, E.: Application of datadriven evidential belief functions to prospectivity mapping for aquamarine-bearing Pegmatites, Lundazi District, Zambia, Natural Resources Research, 14, 47-63, doi:10.1007/s11053-0054678-9, 2005.

Carranza, E., Hale, M., and Faassen, C.: Selection of coherent deposit-type locations and their application in data-driven mineral prospectivity mapping, Ore Geol. Rev., 33, 536-558, doi:10.1016/j.oregeorev.2007.07.001, 2008.

Cassard, D., Billa, M., Lambert, A., Picot, J.-C., Husson, Y., Lasserre, J.-L., and Delor, C.: Gold predictivity mapping in French Guiana using an expert-guided data-driven approach based on a regional-scale GIS, Ore Geol. Rev., 34, 471-500, doi:10.1016/j.oregeorev.2008.06.001, 2008.
Cheng, Q.: Comparison between two types of multifractal modelling, Math. Geol., 28, 1001-1015, doi:10.1007/BF02068586, 1996.

Cheng, Q.: Multifractal distribution of eigenvalues and eigenvectors from $2 \mathrm{~d}$ multiplicative cascade multifractal fields, Math. Geol., 37, 915-927, doi:10.1007/s11004-005-9223-1, 2005.

Cheng, Q.: Singular mineralization processes and mineral resources quantitative prediction: new theories and methods, Earth Science Frontiers, 14, 42-53, 2007a (in Chinese).

Cheng, Q.: Mapping singularities with stream sediment geochemical data for prediction of undiscovered mineral deposits in Gejiu, Yunnan Province, China, Ore Geol. Rev., 32, 314-324, doi:10.1016/j.oregeorev.2006.10.002, 2007b.

Cheng, Q.: Non-linear theory and power-law models for information integration and mineral resources quantitative assessments, Math. Geosci., 40, 503-532, doi:10.1007/s11004-008-9172-6, 2008.

Cheng, Q. and Agterberg, F.: Fuzzy weights of evidence method and its application in mineral potential mapping, Natural Resources Research, 8, 27-35, doi:10.1023/A:1021677510649, 1999.

Cheng, Q. and Agterberg, F. P.: Singularity analysis of ore-mineral and toxic trace elements in stream sediments, Computat. Geosci., 35, 234-244, doi:10.1016/j.cageo.2008.02.034, 2009.

Cheng, Q., Agterberg, F., and Ballantyne, S.: The separation of geochemical anomalies from background by fractal methods, J. Geochem. Explor., 51, 109-130, doi:10.1016/03756742(94)90013-2, 1994.

Cheng, Q., Agterberg, F., and Bonham-Carter, G.: Fractal pattern integration for mineral potential estimation, Natural Resources Research, 5, 117-130, doi:10.1007/BF02257585, 1996.

Cheng, Q., Xu, Y., and Grunsky, E.: Multifractal power spectrumarea method for geochemical anomaly separation, Natural Resources Research, 9, 43-51, 2000.

Cox, D. and Singer, D.: Mineral deposit models, US Geological Survey Bulletin 1693, 379, 143-161, 1986.

De Wijs, H. J.: Statistics of ore distribution, part I, Geologie en Mijnbouw, 13, 365-375, 1951.

Deidda, R.: Multifractal analysis and simulation of rainfall fields in space, Phys. Chem. Earth. Pt. B., 24, 73-78, doi:10.1016/S14641909(98)00014-8, 1999.

Evertsz, C. and Mandelbrot, B.: Multifractal Measures, in: Chaos and Fractals, edited by: Peitgen, H., Jiirgens, H., and Saupe, D., 922-953, Springer-Verlag, New York, 922-953, 1992.

Falconer, K.: Fractal Geometry: Mathematical Foundations and Applications, 2nd edn., Wiley, 2003.

Fisher, R.: The design of experiments, 9th edn., Macmillan, 1971.

Ford, A. and Blenkinsop, T.: Combining fractal analysis of mineral deposit clustering with weights of evidence to evaluate patterns of mineralization: Application to copper deposits of the Mount Isa Inlier, NW Queensland, Australia, Ore. Geol. Rev., 33, 435450, doi:10.1016/j.oregeorev.2007.01.004, 2008.

Lovejoy, S. and Schertzer, D.: Multifractals, cloud radiances and rain, J. Hydrol., 322, 59-88, doi:10.1016/j.jhydrol.2005.02.042, 2006.

Lovejoy, S. and Schertzer, D.: Scaling and multifractal fields in the solid earth and topography, Nonlin. Processes Geophys., 14, 465-502, doi:10.5194/npg-14-465-2007, 2007. 
Lovejoy, S., Schertzer, D., and Stanway, J.: Direct evidence of multifractal atmospheric cascades from planetary scales down to $1 \mathrm{~km}$, Phys. Rev. Lett., 86, 5200-5203, doi:10.1103/PhysRevLett.86.5200, 2001.

Mandelbrot, B. B.: Fractals: Form, Chance, and Dimension, Freeman, San Francisco, 1977.

Mandelbrot, B. B.: The Fractal Geometry of Nature (updated and augmented edition), Freeman, New York, 1983.

Mandelbrot, B. B.: Multifractal measures, especially for the geophysicist, Pure Appl. Geophys., 131, 5-42, doi:10.1007/BF00874478, 1989.

Nykänen, V.: Radial basis functional link nets used as a prospectivity mapping tool for orogenic gold deposits within the central Lapland greenstone belt, northern Fennoscandian shield, Natural Resources Research, 17, 29-48, doi:10.1007/s11053-008-90620, 2008.

Porwal, A., Carranza, E. J. M., and Hale, M.: Artificial neural networks for mineral-potential mapping: A case study from Aravalli Province, western India, Natural Resources Research, 12, 155171, doi:10.1023/A:1025171803637, 2003.

Porwal, A., Carranza, E., and Hale, M.: Bayesian network classifiers for mineral potential mapping, Computat. Geosci., 32, 1-16, doi;10.1016/j.cageo.2005.03.018, 2006.

Raines, G.: Are fractal dimensions of the spatial distribution of mineral deposits meaningful?, Natural Resources Research, 17, 8797, doi:10.1007/s11053-008-9067-8, 2008.

Sahoo, N. R. and Pandalai, H. S.: Integration of sparse geologic information in gold targeting using logistic regression analysis in the Hutti-Maski Schist belt, Raichur, Karnataka, India - a case study, Natural Resources Research, 8, 233-250, doi:10.1023/A:1021698115192, 1999.

Schertzer, D. and Lovejoy, S.: Physical modelling and analysis of rain and clouds by anisotropic scaling of multiplicative processes, J. Geophys. Res., 92(D8), 9693-9714, doi:10.1029/JD092iD08p09693, 1987.
Schertzer, D. and Lovejoy, S.: Nonlinear geodynamical variability: multiple singularities, universality and observables, in: Nonlinear Variability in Geophysics, edited by: Schertzer, D. and Lovejoy, S., Kluwer Academic Publishers, Netherlands, 41-82, 1991.

Singer, D. and Kouda, R.: Application of a feedforward neural network in the search for kuroko deposits in the Hokuroku District, Japan, Math. Geol., 28, 1017-1023, doi:10.1007/BF02068587, 1996.

Turcotte, D.: Fractals and Chaos in Geology and Geophysics, 2nd edn., Cambridge University Press, Cambridge, 1996.

$\mathrm{Xu}, \mathrm{Y}$. and Cheng, Q.: A fractal filtering technique for processing regional geochemical maps for mineral exploration, Geochem.Explor. Env. A., 1(2), 147-156, 2001.

Yu, C., Tang, Y., Shi, P., and Deng, B. L.: The Dynamic System of Endogenic Ore Formation in Gejiu Tin-Polymetallica Ore Region, Yunnan Province, China University of Geosciences, Wuhan, 1988.

Zhang, S., Cheng, Q., and Chen, Z.: Omnibus weights of evidence method implemented in GeoDAS GIS for information extraction and integration, J. China, Univ. Geosci., 19, 404-409, 2008.

Zhang, Z., Mao, H., and Cheng, Q.: Fractal geometry of element distribution on mineral surfaces, Math. Geol., 33, 217-228, doi:10.1023/A:1007587318807, 2001.

Zuo, R., Cheng, Q., Agterberg, F., and Xia, Q.: Application of singularity mapping technique to identify local anomalies using stream sediment geochemical data, a case study from Gangdese, Tibet, western China, J. Geochem. Explor., 101, 225235, doi:10.1016/j.gexplo.2008.08.003, 2009a.

Zuo, R., Cheng, Q., Xia, Q., and Agterberg, F.: Application of fractal models to distinguish between different mineral phases, Math. Geosci., 41, 71-80, doi:10.1007/s11004-008-9191-3, 2009b. 